\title{
SAR ICE IMAGE CLASSIFICATION USING PARALLELEPIPED CLASSIFIER BASED ON GRAM-SCHMIDT SPECTRAL TECHNIQUE
}

\author{
*A.Vanitha ${ }^{1}$, Dr.P.Subashini ${ }^{2}$ and Ms.M.Krishnaveni ${ }^{3}$ \\ ${ }^{1}$ M.Phil Scholar, ${ }^{2}$ Associate Professor, ${ }^{3}$ Research Assistant \\ Department of Computer Science \\ Avinashilingam Institute for Home Science and Higher Education for Women \\ University, Coimbatore, India. \\ *vani.jan23 @gmail. com
}

\begin{abstract}
Synthetic Aperture Radar (SAR) is a special type of imaging radar that involves advanced technology and complex data processing to obtain detailed images from the lake surface. Lake ice typically reflects more of the radar energy emitted by the sensor than the surrounding area, which makes it easy to distinguish between the water and the ice surface. In this research work, SAR images are used for ice classification based on supervised and unsupervised classification algorithms. In the pre-processing stage, Hue saturation value (HSV) and Gram-Schmidt spectral sharpening techniques are applied for sharpening and resampling to attain highresolution pixel size. Based on the performance evaluation metrics it is proved that GramSchmidt spectral sharpening performs better than sharpening the HSV between the boundaries. In classification stage, Gram-Schmidt spectral technique based sharpened SAR images are used as the input for classifying using parallelepiped and ISO data classifier. The performances of the classifiers are evaluated with overall accuracy and kappa coefficient. From the experimental results, ice from water is classified more accurately in the parallelepiped supervised classification algorithm.
\end{abstract}

\section{KEYWORDS}

SAR Ice classification, Gram-Schmidt spectral sharpening, supervised classification, unsupervised classification, Kappa coefficient.

\section{INTRODUCTION}

Synthetic aperture radar (SAR) is a microwave sensor which is capable of producing highresolution images of the earth's surface. A SAR uses a transmitter and passing out a microwave signal, and a receiver collects that pulse reflected back to the satellite. Radar wavelengths are much longer than those of visible or infrared light, and, for this reason, a SAR can see through cloud, smoke fog and moisture area; it can take images in day or night; and depending on the signal frequency [1]. SAR has two fundamental dimensions and three modes. The slant range 
and azimuth are the dimensions used for target discrimination in range involve the correlation of each echo with the corresponding transmitted pulse. An increase of the swath width can also be made at the expense of azimuth resolution, by interrupted switching of the radar beam between adjacent sub-swaths. Medium to low resolution images can be obtained using this ScanSAR imaging mode [2].

Image enhancement is making improvement in satellite image quality without knowledge about the source of degradation. Image sharpening tools are used to automatically merge a lowresolution colour, multi-, or hyper-spectral image with a high-resolution gray scale image [3].Image classification is probably the most important part of image analysis. SAR ice images are classified using two main classification methods namely supervised and unsupervised classification algorithms [8]. The performance of the classifiers depends upon the overall accuracy and kappa coefficients. At present it is not possible to state which classifier is best for all situations as the characteristics of each image and the circumstances for each study vary so greatly. In this paper, Image enhancement is described in the second section, classification of SAR image is explained in the third section, and the experimental results and conclusion are discussed in fourth section.

\section{IMAGE ENHANCEMENT}

Image enhancement is the enhancement of an original image appearance by increasing dominance of some features or by decreasing ambiguity between different regions of the image. In this research work, image sharpening technique is applied and the purpose of such an image sharpening method is to increase sharpness of images. Image sharpening refers to enhancement technique that highlights edges and fine details in an image. Sharpened images provide clear image to view visually and assist for the classification. Hue Saturation Value (HSV) and GramSchmidt spectral sharpening techniques are two method used for image sharpening [3].

\subsection{Hue Saturation Value (HSV) sharpening}

HSV sharpening used to transform an RGB image to HSV color space and replace the value band with the high-resolution image. The HSV techniques automatically resample the hue and saturation bands to the high-resolution pixel size using a nearest neighbour technique and finally transform the images back to RGB color space. The output of the original images will have the high pixel size with the input of high-resolution data. The subjective evaluation of the HSV sharpening technique is shown in Fig: 1.

\subsection{Gram -Schmidt spectral sharpening}

Gram-Schmidt spectral sharpening is used to sharpen multispectral data using high spatial resolution data. First, a panchromatic band of the original image is simulated from the lower spatial resolution spectral bands. Secondly, a Gram-Schmidt transformation technique is performed on the simulated panchromatic and the spectral bands, where the simulated panchromatic band of the images are taken as the first band. Then, the first Gram-Schmidt band is swapped with the high spatial resolution panchromatic band. At the end, inverse Gram-Schmidt transform is then applied to images and form the sharpened spectral bands. 
Computer Science \& Information Technology (CS \& IT)

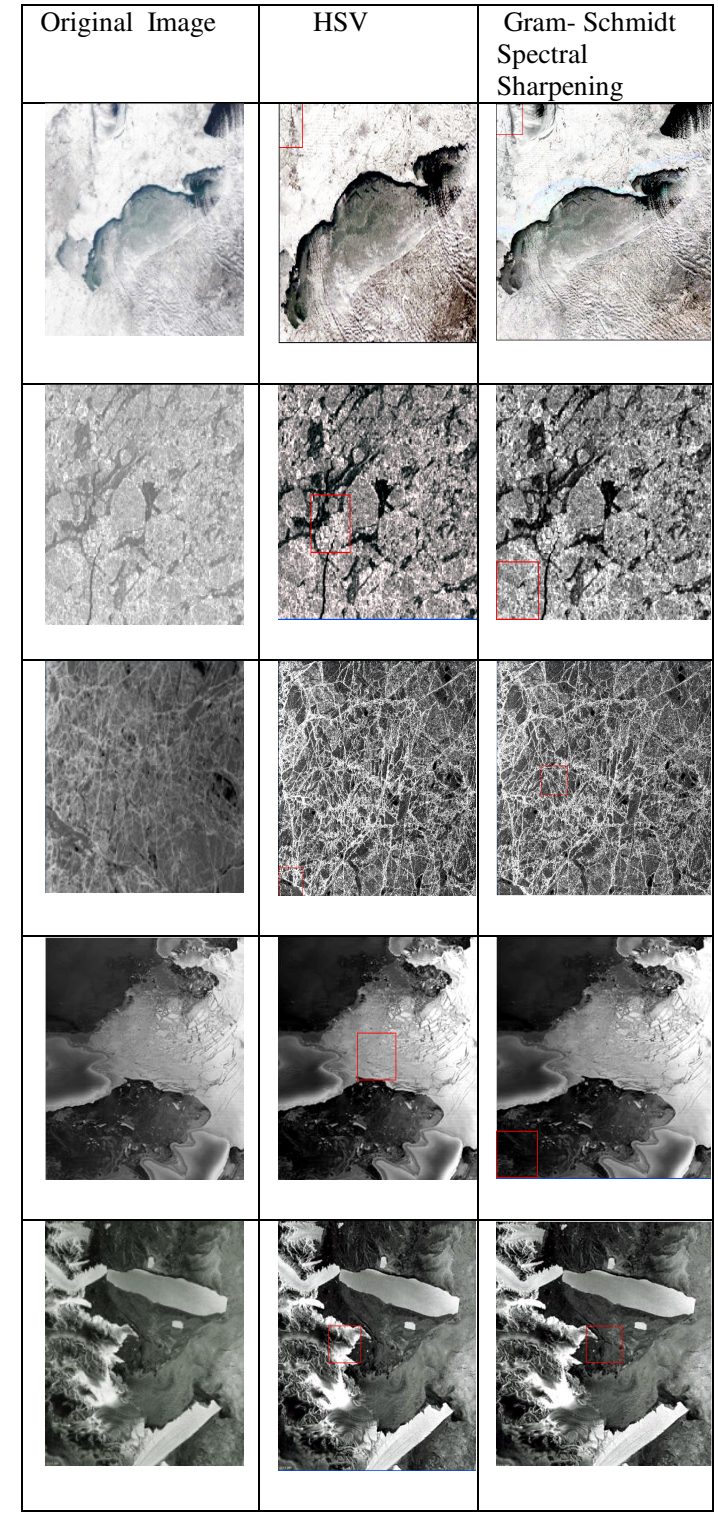

Fig 1: Image Sharpening result for HSV and Gram-Schmidt spectral sharpening

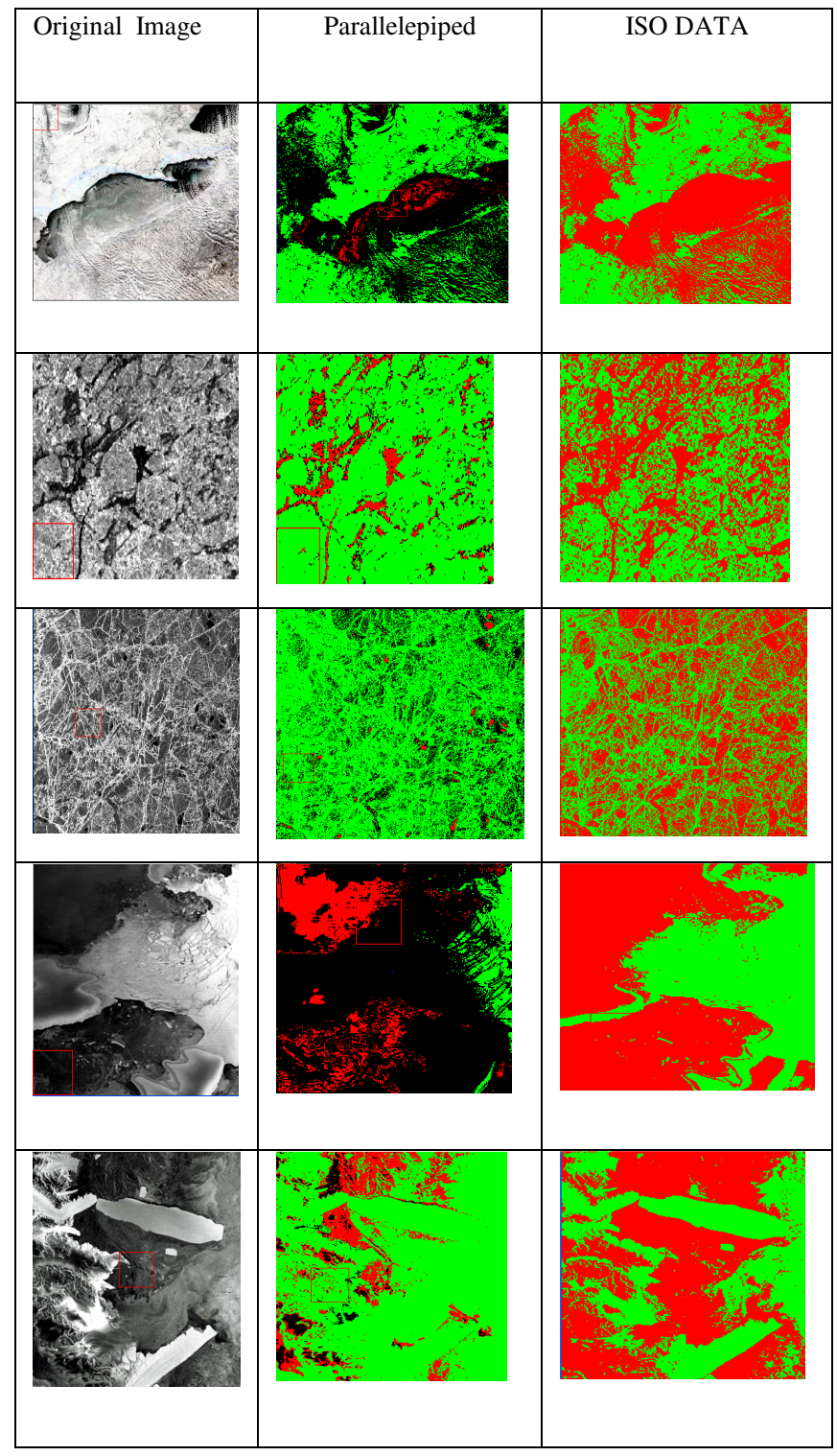

Fig 2: Classification result for supervised a Unsupervised classifier

\section{Classification AlgorithmS}

The water and the ice are classified from SAR images by using supervised and unsupervised classification algorithms.

\subsection{Supervised classification}

Supervised classification is used to cluster pixels in images into classes corresponding to user defined training classes. In this research, parallelepiped classifier is taken for the water and ice classification. The supervised classifier use the ground truth ROI image as training set for 
classifying the class and the overall accuracy and kappa coefficient is calculated from the parallelepiped classified image and ground truth ROI [10].

\subsubsection{Parallelepiped classifier}

Parallelepiped classification uses a simple decision rule to classify SAR images. The decision boundaries form an n-dimensional parallelepiped classification in the image data space. In the parallelepiped classification, dimensions are defined based upon a standard deviation threshold from the mean of each selected class. If a pixel value images lies above the low threshold and below the high threshold for all bands being classified, it is assigned to that class. Area that do not fall within any of the trained pixel then they are designated as unclassified [10].

\subsection{Unsupervised classification}

Unsupervised classification is comparable to cluster analysis where interpretations are assigned to the same class because they have related values. In this work, ISODATA classifier is taken for classifying the water and ice surface in the lake area. The unsupervised classification doesn't need any training area for the classification [7].

\subsubsection{ISODATA Classifier}

The ISODATA (Iterative Self-Organizing Data Analysis Technique) Classification method uses an iterative approach that incorporates a number of heuristic procedures to compute classes. The ISODATA classification method is similar to the K-Means method, but incorporates measures for splitting and combining the trial classes to obtain an optimal set of output classes. In ISO data classifier, user has to assign the number of classes and it classifies according to the user defined classes [7].

\section{EXPERIMENTAL RESULTS}

The above assessment techniques are implemented on SAR images. Fig.1 and Fig.2 shows the results of sharpening technique and classification algorithms in subject evaluation. Hence, this work is an attempt to study the ice classification and to evaluate the performance of supervised and unsupervised algorithms.

\subsection{Results of Image Enhancement}

Fig.1 shows the subject evaluation results of sharpening technique. By comparing the error rate of sharpening techniques metrics, the resultant images of Gram-Schmidt spectral sharpening method appears with high resolution. So, Gram-Schmidt spectral sharpening images are taken as input for the classification. The tables $1,2,3,4$ and 5 shows the metrics used in image sharpening techniques, to identify which techniques gives better results.

\section{Entropy (En)}

The Entropy of an image is a measure of information content but has not been used to assess the effects of information change in SAR images. Entropy reflects the capacity of the information carried by SAR images. The error rate of entropy should be high and that have high information in the images. The formula used to calculate the Entropy is 


$$
\mathrm{En}=-\sum_{i=0}^{255} \mathrm{P}(\mathrm{i}) \log _{2} \mathrm{P}(\mathrm{i})
$$

where, $\mathrm{P}$ (i) is the ratio of the number of the pixels with gray value equal to $\mathrm{i}$ over the total number of the pixels [4].

\section{Root mean square Error (RMSE)}

The RMSE is a quadratic scoring rule which measures the average magnitude of the error. The corresponding experimental values are each squared and then averaged over the sample images. Finally, the square root of the average is taken in the SAR images. Since the errors are squared before they are averaged, the RMSE gives large errors by increasing weight. The formula used to calculate the RMSE is

$$
R M S=\sqrt{\frac{\sum_{i=1}^{n} X_{i}^{2}}{n}}
$$

where, $\mathrm{n}$ denotes discrete distribution $x_{i}$ and $X_{i}^{2}$ denotes the mean square values.

\section{Correlation Coefficient (CC)}

The correlation coefficient measures the closeness or similarity between two images. It can vary between -1 to +1 . A value close to +1 indicates that the two images are much related, while a value close to -1 . The formula to compute the correlation is

$$
r=\frac{\left.n\left(\sum x y\right)-\left(\sum x\right)\left(\sum y\right)\right)}{\sqrt{\left.\left[n \sum x^{2}-\left(\sum x\right)^{2}\right)\right]\left[n \sum y^{2}-\left(\sum y\right)^{2}\right]}}
$$

where, $\mathrm{n}$ denotes number of values or elements and then the $\mathrm{x} \& \mathrm{y}$ are the two variables of correlation coefficient. [4].

\begin{tabular}{|c|c|c|}
\hline $\begin{array}{c}\text { SAR } \\
\text { ICE } \\
\text { Image }\end{array}$ & HSV & $\begin{array}{c}\text { Gram- Schmidt } \\
\text { Spectral } \\
\text { Sharpening }\end{array}$ \\
\hline Image 1 & 7.1872 & 7.2541 \\
\hline Image 2 & 4.4695 & 4.5301 \\
\hline Image3 & 4.5412 & 4.5879 \\
\hline Image 4 & 4.6457 & 4.7001 \\
\hline Image 5 & 4.6891 & 4.7874 \\
\hline
\end{tabular}

\begin{tabular}{|c|c|c|}
\hline $\begin{array}{c}\text { SAR } \\
\text { ICE } \\
\text { Image }\end{array}$ & HSV & $\begin{array}{c}\text { Gram- Schmidt } \\
\text { Spectral } \\
\text { Sharpening }\end{array}$ \\
\hline Image 1 & 34.87182 & 34.44457 \\
\hline Image 2 & 32.00580 & 31.41486 \\
\hline Image3 & 32.86569 & 32.01721 \\
\hline Image 4 & 34.45883 & 34.09934 \\
\hline Image 5 & 32.67526 & 32.40524 \\
\hline
\end{tabular}


Table .1 Results based on Entropy matrices

Table .2 Results based on RMSE matrices

\begin{tabular}{|c|c|c|}
\hline $\begin{array}{c}\text { SAR ICE } \\
\text { Image }\end{array}$ & HSV & Gram- Schmidt Spectral Sharpening \\
\hline Image 1 & 0.5820497620 & 0.5676583494 \\
\hline Image 2 & 0.5784706080 & 0.5771488506 \\
\hline Image3 & 0.9678437137 & 0.9770377594 \\
\hline Image 4 & 0.5930112531 & 0.5947986552 \\
\hline Image 5 & 0.6789349664 & 0.8769786350 \\
\hline
\end{tabular}

Table .3 Results based on Correlation Coefficient (CC) matrices

\section{Universal image quality index}

The two images are considered as matrices with $M$ column and $N$ rows containing pixel values, respectively. The universal image quality index $Q$ may be calculated as a product of three components namely loss of correlation, luminance distortion, and contrast distortion. The value range for this component is also $[0,1]$.The formula used to calculate universal image quality index is

$$
\mathrm{Q}=\frac{4 \sigma_{x y} \bar{x} \bar{y}}{\left(\sigma_{x}^{2}+\sigma_{y}^{2}\right) \llbracket(\bar{x})^{2}+(\bar{y})^{2} \rrbracket}
$$

where $\mathrm{Q}$ refers to quality index, $\bar{x} \bar{y}$ denotes mean of original image $\mathrm{x}$ and testing image $\mathrm{y}, \sigma_{x}^{2}$ refers to variance of original image.

\begin{tabular}{|l|l|l|}
\hline $\begin{array}{l}\text { SAR } \\
\text { ICE } \\
\text { Image }\end{array}$ & HSV & $\begin{array}{l}\text { Gram- } \\
\text { Schmidt } \\
\text { Spectral } \\
\text { Sharpening }\end{array}$ \\
\hline Image 1 & 0.59370 & 0.53829 \\
\hline Image 2 & 0.80670 & 0.81128 \\
\hline Image3 & 0.86944 & 0.84957 \\
\hline Image 4 & 0.40543 & 0.39688 \\
\hline Image 5 & 0.53491 & 0.51712 \\
\hline
\end{tabular}

Table .4. Results based on Universal image quality index matrices

\begin{tabular}{|c|l|l|}
\hline $\begin{array}{l}\text { SAR ICE } \\
\text { Image }\end{array}$ & HSV & $\begin{array}{l}\text { Gram- } \\
\text { Schmidt } \\
\text { Spectral } \\
\text { Sharpening }\end{array}$ \\
\hline Image 1 & 6.47217 & 6.40362 \\
\hline Image 2 & 6.39154 & 6.27559 \\
\hline Image3 & 6.44182 & 6.40635 \\
\hline Image 4 & 6.45976 & 6.53826 \\
\hline Image 5 & 6.40324 & 6.29785 \\
\hline
\end{tabular}

Table .5 Results based on MAE matrices

\section{Mean absolute Error (MAE)}

The Mean Absolute Error measures the average magnitude of the errors in a set of forecasts, without considering their direction. It measures accuracy for continuous variables. The MAE is a 
linear score which means that all the individual differences are weighted equally in the average. The formula used to calculate the MAE is

$$
\mathrm{MAE}=\frac{1}{n} \sum_{i=0}^{n}\left|e_{i}\right|
$$

where, the mean absolute error is an average of the absolute errors $e_{i}=f_{i}-y_{i}$, where $f_{i}$ is the prediction and $\mathrm{y}_{\mathrm{i}}$ the true value.

In above results, error rate of the entropy and universal image quality index has the high error rate and RMSE, correlation coefficient and MAE has the low error rate in the Gram-Schmidt spectral sharpening compared to HSV. By comparing the error rate of metrics and objective evaluation of Gram-Schmidt spectral sharpening technique show the better result for sharpening boundaries.

\subsection{Classification algorithms Result}

Fig.2,3 and 4 shows the subjective evaluation and accuracy assessment results of supervised and unsupervised classification algorithms. The overall accuracy and kappa coefficient are calculated from classified images and ground truth ROI images.

The accuracy assessment generated from the parallelepiped supervised classification technique showed an overall classification accuracy was $80.43 \%$ with Kappa statistic of 0.69 , but in the unsupervised classification technique, the overall accuracy decreased to $75.03 \%$ with Kappa statistic of $0.62 \%$.By comparing the overall accuracy with kappa statistic parallelepiped supervised classifier perform better then ISO data classifier.

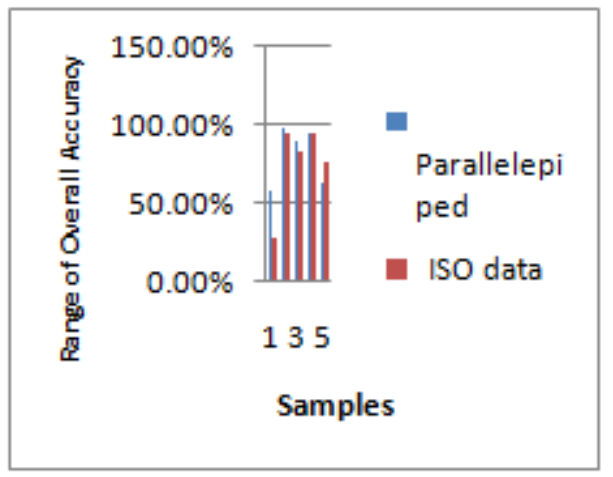

Fig. 3. Performance evaluation of the classifiers based on overall accuracy

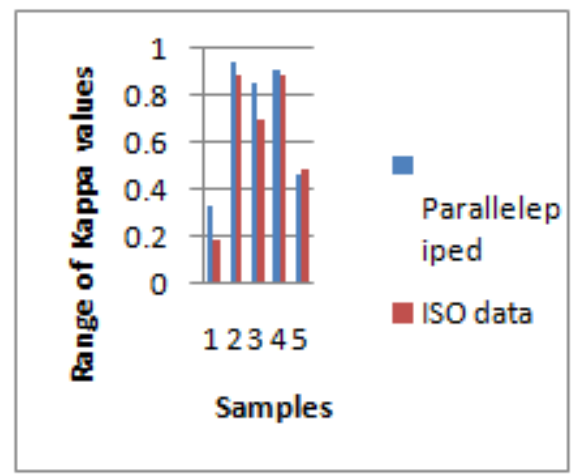

Fig. 4. Performance evaluation of the classifiers based on kappa coefficients

\section{CONCLUSION}

In this paper, the ice classification from the lake surface was implemented by unsupervised and unsupervised classification algorithms. In the classification process, the SAR ice images are enhanced using HSV and Gram-Schmidt spectral sharpening techniques. By comparing metrics results of the sharpening techniques, Gram-Schmidt sharpening method performs better than the HSV between the boundaries. The ice classification algorithms classify the water and the ice surface in the lake area and accuracy assessments of the algorithms are assessed using overall 
accuracy and kappa coefficient. As the result parallelepiped supervised classification appears more accurate than the ISO data unsupervised classification. In future, types of ices classification will be classified by extracting features of ice and implementing the segmented methods for SAR images.

\section{REFERENCES}

[1] Hacene Akliouat, Youcef Smara and Lynda Bouchemakh, (2007) "Synthetic aperture radar image formation process: application to a region of north Algeria" Proc. 'Envisat Symposium 2007', PP 23-27.

[2] Müjdat Çetin, and William Clem Karl, (2001) "Feature-Enhanced Synthetic Aperture Radar Image Formation Based on Nonquadratic Regularization" IEEE TRANSACTIONS ON IMAGE PROCESSING, VOL. 10, NO. 4,PP 623-631.

[3] Raman Maini and Himanshu Aggarwal,( 2010) "A Comprehensive Review of Image Enhancement Techniques" Journal of computing, volume 2, issue 3, ISSN 2151-9617, ,PP 8-13.

[4] Firouz Abdullah Al-Wassail and Dr. N.V. Kalyankar, (2012) "A novel metric approach evaluation for the spatial enhancement of pansharpene images" International Conference of Advanced Computer Science \& Information Technology pp. 479-493.

[5] P. Mishra, Y. Yamaguchi and D. Singh, (2011) "Land cover classification of palsar images by knowledge based decision tree classifier and supervised classifiers based on sar observables" Progress In Electromagnetics Research B, Vol. 30,PP 47-70.

[6] Komal Vij and Yaduvir Singh"Enhancement of Images Using Histogram Processing Techniques" International. Journal of coputer Technology Application., Vol 2 (2),PP 309-313.

[7] Mohd Hasmadi I, Pakhriazad HZ, Shahrin MF, (2009) " Evaluating supervised and unsupervised techniques for land cover mapping using remote sensing data "Malaysian Journal of Society and Space 5 issue, ISSN 2180-2491,PP 1-10.

[8] C. Palaniswami, A. K. Upadhyay and H. P. Maheswarappa., (2006) "Spectral mixture Analysis for sub pixel classification of coconut", Current Science, Vol. 91, No. 12, pp. 1706 -1711.

[9] N. Roshani , M. J. Valadan Zouj , Y. Rezaei , M.Nikfar,( 2008) " Snow mapping of alamchal glacier using remote sensing data" The International Archives of the Photogrammetry, Remote Sensing and Spatial Information Sciences. Vol. XXXVII.PP 804-808.

[10] Perumal and R.Bhaskaran, (2010) "Supervise Classification performance of multispectral images", Journal of Computing, Volume 2, PP 124-129.

[11] P.M.Atkinson and A.R.L.Tatnal, (1997) "Introduction to neural networks in remote sensing", International Journal of Remote sensing, Volume 11, pp. 699-709.

[12] Daniel Gomez and Javieer Montero(2011) " Determining the accuracy in image supervised classification problems " EUSFLAT-LFA Aix-les-Bains, France,PP 342-349.

[13] Xlangming Xiao,Zhenxi shen and Xieoguan Qin (2001)" Assessing the potential of vegetation sensor data for mapping snow and ice civer a normalized difference snow and ice index" International Journal Remote sensing, Volume 22,pp.2479-2487.

[14] Jorge Haarpaintner, (2002) "Sea ice classification of ers-2 sar images based on a nested-correlation ice-tracking algorithm", 16th IAHR International Symposium on Ice, Dunedin, New Zealand.

[15] Limin Jiao and Yaolin Liu., (2012) "Analyzing the shape characteristics of land use classes in remote sensing imagery”,XXII ISPRS Congress, Volume 1-7. 